\title{
liver $X$ receptors inhibit pulmonary inflammation and airway remodeling induced by ozone exposure in mice
}

\section{Fenfang Yu}

Nanjing First Hospital

Lin Zheng

Nanjing First Hospital

Li Wang

Nanjing First Hospital

Xiaobing Yang

Nanjing First Hospital

Zhifei Ma

Nanjing First Hospital

Yu Tang

Nanjing First Hospital

Nan Li

Nanjing First Hospital

Youran Wang

Nanjing First Hospital

Wei Gu

Nanjing First Hospital

Ying Shi ( $\nabla$ sykxss@163.com )

Nanjing First Hospital https://orcid.org/0000-0003-3734-9890

Research

Keywords: Ozone, Liver X receptors (LXRs), Inflammation, Airway remodeling

Posted Date: June 22nd, 2020

DOI: https://doi.org/10.21203/rs.3.rs-35955/v1

License: (c) (i) This work is licensed under a Creative Commons Attribution 4.0 International License.

Read Full License 


\section{Abstract}

Ozone $\left(\mathrm{O}_{3}\right)$ exposure can lead to airway inflammation, hyperreactivity and airway remodeling. Liver $\mathrm{X}$ receptors (LXRs) play an important role in attenuating inflammation. The therapeutic effects of LXRs on ozone-induced pulmonary inflammation and airway remodeling were examined. C57/BL6 mice were exposed to ozone for 1 or 6 weeks. LXR agonist T0901317 was administered to mice at one hour before ozone exposure. As expected, ozone-exposed mice developed lung inflammation with augmented neutrophil, macrophage, TNF-a, IL-6, IL-8 and G-CSF in the bronchoalveolar lavage fluid and serum. The increase in MMP-9 and a-SMA expression, and collagen deposition around airway reflected the airway remodeling in ozone-exposed mice. Compared with ozone-exposed mice, LXR agonist T0901317 inhibited the ozone-induced inflammation after 1 and 6 weeks in the ozone exposure model. In addition, the treatment with the LXR agonist prevented alveolar enlargement and reduced airway remodeling in 6-week ozone-induced mice. Therefore, LXRs may repress pulmonary inflammation and attenuate the progression of airway remodeling.

\section{Background}

Ozone is a common air pollutant, which causes a variety of human adverse health issues, especially respiratory diseases. Inhalation of ozone has a significant effect on decreased lung function and airway inflammation. Some clinical studies have confirmed that exposure to high levels of ozone can lead to worsened symptoms and increased hospitalization rate for patients with asthma[1]and COPD[2]. Ozone has been used in a variety of animal species. It can induce lung inflammation accompanied by airway remodeling[3, 4].

The lung ling fluid (including the trachea, bronchi and alveolar) is rich in $90 \%$ lipid and 10\% protein. Most of the lipids are unsaturated fatty acids, especially monounsaturated fatty acids[5]. Inhaled ozone can easily react to the lung ling fluid and form a lipid ozonation product (LOP), which might be related to the pro-inflammatory effect of ozone. In the human airway epithelium, LOP can activate pro-inflammatory factors, such as IL-6 and IL-8[6, 7]

Liver $X$ receptors (LXRs) are transcription factors belonging to the nuclear receptor superfamily and have two isoforms: $a(\mathrm{NR} 1 \mathrm{H} 3)$ and $\beta(\mathrm{NR} 1 \mathrm{H} 2)$ [8]. When combined with natural ligands (e.g. oxysterols and desmosterol)[9] or synthetic ligands (e.g. GW3695 and T0901317)[10, 11], LXRs play a vital role in cholesterol homeostasis and repressing inflammation. Activated LXRs regulate the transcription of genes involved in cholesterol efflux and macrophage-mediated inflammation. Therefore, LXRs play an antiinflammatory role in atherosclerosis[12, 13]. In addition, it has been confirmed that synthetic LXR agonist T0901317 can attenuate lipopolysaccharide (LPS)-induced acute lung injury in rats[14]. However, the effect of the LXR agonist on pulmonary inflammation and airway remodeling induced by ozone exposure have not been elucidated. 
It was hypothesized that the LXR agonist might have therapeutic potential for ozone-induced lung inflammation and airway remodeling. Considering the different times and frequency of ozone exposure, which led to different lung injuries and inflammation[15], the effects of LXRs on different period of ozoneinduced lung inflammation and airway remodeling in mice were investigated.

\section{Materials And Methods}

\section{Exposure to ozone}

Ten-week old pathogen free C57/BL6 male mice were raised under suitable conditions. These mice were exposed to ozone produced by an ozonizer (Sander Ozonizer, Germany) mixed with air for three hours at a concentration of 2.5 parts per million (ppm) in a glass container. The ozone concentration was continuously monitored using an ozone probe (Analytical Technology, UK). The mice exposed to ozone were divided into four groups: (1) exposure for three hours every day, continued for seven days; (2) exposure for three hours every day, for over six weeks; the ozone exposure time and frequency of (3) and (4) were the same as those of (1) and (2), respectively. The difference was that the LXR agonist (T0901317, Sigma-Aldrich, USA, $30 \mathrm{ml} / \mathrm{kg}$ ) needed to be intraperitoneally injected at one hour before ozone exposure. Control animals were exposed to air equivalent time. Mice were sacrificed at 24 hours after last exposure to ozone.

\section{Blood collection}

After anesthetizing mice, blood was collected. Then, mice were killed using the dislocated method. The collected blood was left in the centrifuge tube at room temperature for two hours, and centrifuged at $4,000 \mathrm{rpm}$ for 10 minutes. Then, the supernatant was moved into a clean $1.5-\mathrm{ml}$ centrifuge tube and stored at $-80^{\circ} \mathrm{C}$.

\section{Bronchoalveolar lavage fluid (BALF)}

Mice were sacrificed, and tracheotomy was performed. In order to obtain the BALF, ice-cold PBS (0.5 ml) was infused into the lungs and withdrawn via tracheal cannulation, thrice (total volume: $1.5 \mathrm{ml}$ ). The total cell count and differential cell counts from the slide and the stains performed by Wright-Giemsa stain (KeyGEN BioTECH, China) were determined using an optical microscope (Olympus Optical, Tokyo, Japan). At least 200 cells were counted for each mouse, and were identified as macrophages, lymphocytes and neutrophils, according to the standard morphology under $\times 400$ magnification. The supernatant obtained from the BALF was stored at $-80^{\circ} \mathrm{C}$ for subsequent biochemical analysis.

\section{Measurement of proinflammatory cytokine levels in BALF and serum}

The levels of IL-6, IL-8, TNFa (KeyGEN BioTECH, China) and G-CSF (MULTI SCIENCES, China) in BALF and serum were quantified by ELISA, according to the manufacturer's protocols. 
After the BALF samples were obtained, the left lung tissue was fixed in 10\% (v/v) neutral buffed formalin. Then, the tissue was embedded in paraffin, sectioned at 5-um thickness, and stained with hematoxylin and eosin (H\&E) solution to estimate the inflammation response. Then, the sections were stained using Masson Trichrome staining to identify the collagen deposition, according to the standard protocol recommended by the manufacturer. All histologic examinations were performed in a double blinded manner under $400 \times$ magnification.

\section{Inflammation score}

The investigators identified 3-4 medium-sized bronchus with a diameter of 60-125 microns. The pathological changes of the alveolar hemorrhage, interstitial hyperemia, infiltration of inflammatory cells between and around the alveolar, and thickness of the alveolar membrane or formation of the hyaline membrane were observed under a 400 -fold microscope. The lesions were classified into five grades: 0 , extremely mild injury; 1 , mild injury; 2 , moderate injury; 3 , severe injury; 4 , severe injury. The scores of the above four indicators were added to obtain the total score.

\section{Mean linear intercept (Lm)}

The H\&E sections were observed under a 200x magnification optical microscope, and 10 peripheral visual fields were randomly taken from each section. The images of the sections were opened in Photoshop, a cross line was drawn through the positive center of the image (avoiding the fields of airways and vessels as much as possible), the number of alveolar spaces $(n)$ that passed through these two lines were counted, and the total length of the cross line $(L)$ was measured, which was calculated using the formula, $\mathrm{Lm}=\mathrm{L} / \mathrm{N}$. The value represents the average alveolar diameter, reflects the degree of alveolar damage[16].

\section{Immunostaining for a-SMA and MMP9}

The paraffin slices are dewaxed in water. Then, these were incubated in $3 \% \mathrm{H}_{2} \mathrm{O}_{2}$ at room temperature for 10 minutes to eliminate the activity of the endogenous peroxidase. Afterwards, these were rinsed with distilled water, and soak in PBS for five minutes, twice. Afterwards, these were closed with $5 \%$ normal goat serum (diluted with PBS), incubated at room temperature for 10 minutes, poured out to the serum, and were not washed. Next, the anti-MMP9 (Servicebio GB11132) and anti-a-SMA (Servicebio GB13044) solutions were added, and the slices were incubated for two hours at $37^{\circ} \mathrm{C}$, or overnight at $4^{\circ} \mathrm{C}$. Then, these were rinsed with PBS for five minutes, for three times. Afterwards, the appropriate HRP-labeled secondary antibody working solution (Servicebio GB23303) was added, the slices were incubated at $37^{\circ} \mathrm{C}$ for 30 minutes, and rinsed with PBS for five minutes, for three times. Subsequently, an appropriate amount of horseradish enzyme-labeled streptomycin working solution was added, and the slices were incubated at $37^{\circ} \mathrm{C}$ for 30 minutes. Then, these were rinsed with PBS for five minutes, for three times. The Chromogenic agent was applied for eight minutes. Full flushing, re-dyeing, dehydration, transparency and sealing of tap water were performed. 


\section{Oil red 0 assay}

The BALF from mice was extracted and plated on slides with DMEM (Solarbio). The cells were incubated for four hours to allow for adhesion, washed for three times with sterile PBS, added with $4 \%$ paraformaldehyde fixative solution, and fixed for 20 minutes. Then, these cells were washed for three times with pure water, and the slides were dripped with $60 \%$ isopropanol for 15 seconds. Next, the Oil Red $O$ stain solution (KeyGEN BioTECH, China) was added to each slide, and these were incubated for 30 minutes at $37^{\circ} \mathrm{C}$. After 30 minutes, these were washed with $60 \%$ isopropanol for 15 seconds (with strict control of the time). Then, these cells were washed with pure water for three times, added with the hematoxylin dye solution for five minutes, carried on a water-based sealing film, and imaged using a Nikon Eclipse Ti (Nikon, Tokyo, Japan). The results were analyzed by taking the percentage of the stained cells over the total cell number of cells counted.

\section{Statistical analysis}

The data are presented as mean \pm standard error of the mean. The statistical differences among the control air group, and 1- and 6-weeks exposure group with or without the LXR agonist were analyzed by one-way ANOVA (with nonparametric or mixed test) with the multiple comparison test for all data (GraphPad Prism 8.0). A $P$-value of $<0.05$ was considered statistically significant.

\section{Results}

\section{LXR agonist decreases the total and differential cell count of BALF}

After mice exposure to ozone, the increase in total cells in the BALF was mainly manifested by the increase in neutrophils, macrophages and lymphocytes. These changes are more pronounced with the prolonged ozone exposure (Fig. 1). The LXR agonist significantly reduced the cell count in ozone-induced BALF, especially the macrophages and neutrophils (Fig. 1).

\section{LXR agonist inhibits the inflammatory infiltration in lung tissue and cytokines in BALF and serum}

Compared with ozone exposure controls, LXR-treated mice exposed to ozone exhibited a decrease in IL-6, TNF- $\alpha$ and G-CSF in the BALF and serum. In addition, the LXR agonist significantly decreased the level of IL-8 in the BALF, but there was no significant difference in serum (Figs. $2 A$ and $2 B$ ). The H\&E staining of lung tissues was also performed to obtain the inflammation score. The results revealed that the LXR agonist can inhibit the infiltration of inflammatory cells in lung tissues (Figs. 2C-2H).

\section{LXR agonist reduces $L m$ and inhibits the alveolar enlargement}

The mean linear intercept (Lm) significantly increased in 6-week ozone-exposed mice, when compared to air-exposed mice, and this also slightly increased in 1-week ozone-exposed mice. The slight difference in outcome from a previous study [17]was possibly because in the present study, mice were more frequently exposed to ozone. The treatment with the LXR agonist prevented the alveolar enlargement that 
manifested as lower Lm in 6-week ozone-induced mice. However, this effect was not obvious in mice exposed to ozone for one week. (Fig. 3).

\section{LXR agonist attenuates the collagen deposition associated with ozone exposure}

The effect of LXR agonist T0901317 on bronchial fibrosis was explained by the Masson's staining analysis of lung tissues (Fig. 4). Compared with the control group, the ozone exposure increased the deposition of fibers around the bronchus, and this became more obvious with the increase in ozone exposure time (Figs. 4B and 4D). However, the LXR agonist treatment significantly reduced the lung collagen levels (Figs. 4C and 4E).

\section{LXR agonist reduces MMP9 expression and a-SMA generation in lung tissues}

The investigators examined the diverse markers that reflect airway remodeling features, in order to investigate the role of the LXR agonist in ozone-induced airway remodeling, including a-SMA and MMP9. It was found that ozone inhalation markedly increased the a-SMA generation and MMP9 expression in lung tissues, especially in the 6-week ozone exposure model (Figs. 5Ab and 5Ad). The LXR agonist treatment attenuated the MM9 expression relative to untreated 6 week-ozone exposure mice (Fig. $5 \mathrm{Ae}$ ). However, the inhibition was not significant in mice exposed to ozone for one week (Fig. 5Ac). There was a similar inhibition of a-SMA generation after treatment with the LXR agonist in lung tissues (Fig. 5B).

\section{Ozone exposure does not lead to the formation of foam macrophages}

Studies have shown that the LXR agonist can significantly reduce the number of alveolar foam macrophages by Oil red $\mathrm{O}$ staining in a chronic cigarette smoke exposure model[18]. Since there are some similarities between the ozone model and smoking model, Oil Red $\mathrm{O}$ staining was used to determine whether ozone can cause the formation of foam macrophages. However, the result was not consistent with the expectations of the investigators, and foam macrophages were not found in the BALF in the experimental group (Fig. 6).

\section{Discussion}

Although growing evidence has supported that the activation of LXRs contribute to the inhibition of inflammation in vivo and in vitro[19-22], the LXRs failed to affect allergic inflammation[22]. So it is important to identify the roles of LXRs in different airway diseases. In the present study, ozone exposure lead to inflammatory cells infiltration in airway accompanied by increased cytokines and chemokines in the BALF and serum. LXR agonist has a certain protective effect on ozone-induced lung inflammation and airway remodeling. Consistent with previous results [17, 23], the number of BALF cells, mainly neutrophils and macrophages significantly increased after ozone exposure. The mechanism of the consequent lung injury and airway remodeling was different with allergic asthma which characterized with eosinophils infiltration. It seems that ozone induced inflammation responds well to LXR ligand treatment. 
TNF-a is a powerful inflammatory factor that can stimulate leukocytes and endothelial cells to express adhesion molecules and upregulate the expression of other inflammatory cytokines, such as IL- 6 and IL$8[24,25]$. In the paraquat-induced lung injury model, T0901317 attenuates the TNF-a generation in a dose dependent manner[26]. In addition, the LXR agonist represses the LPS-induced increase in TNF-a level[27]. In the present study, T0901317 markedly decreased the TNF-a level. This contributed to the reduced production of inflammatory factors and chemokines by inflammatory cells.

IL-6 and IL-8 are important chemokines for neutrophils and lymphocytes, which can increase the number of sectional inflammatory cells, promote and aggravate airway inflammation response, and reflect the severity of airway inflammation to some extent. Charles et al. reported the overexpression of IL-6 leads to alveolar enlargement, airway wall thickening, and the infiltration of monocytes around the trachea, suggesting that IL-6 participates in the airway remodeling process of emphysema[28]. LXRs are regulators of inflammatory signaling[20]. Treatment with LXR agonists results in the SUMOylation of LXRs and SUMO-dependent interaction between LXR and CORO2A. The LXR-CORO2A interaction impairs the inflammatory gene expression[29-31]. LXR agonist T0901317 inhibited the 03 induced expression of IL- 6 and IL- 8 in the BALF and serum which contribute to decrease the amount and activity of inflammatory cells. The sebsequent airway remodeling was also attenuated.

G-CSF is a major extracellular regulator of the innate immune system, which influences the survival, proliferation and differentiation of neutrophils[32]. This can lead to the propagation of inflammatory response, airway construction and remodeling. The release of G-CSF was restrained by T0901317-treated human airway smooth muscle (hASM) cells[33]. In our study, after injecting T0901317 into mice, the production of G-CSF was alleviated. the number of neutrophils were correspondingly decreased. These results strongly suggest the LXR agonist T0901317 can inhibit the neutrophil and macrophage-mediated airway inflammation induced by ozone exposure.

It was speculated that sterol transporter ABCA1 might be the key gene for the LXR agonist in its antiinflammatory role. ABCA1 is the target gene of LXR, which can be activated by LXR transcription and plays an important role in cholesterol efflux. ABCA1 mediates the LXR agonist to inhibit the inflammatory gene expression by repressing the TLR-induced NF-KB and MAPK pathway activation[34]. Meanwhile, David G. Thomas considered that cholesterol efflux mediates the LXR anti-inflammatory effects[35]. The future studies should further investigate the anti-inflammation mechanism of ABCA1 in ozone-exposure mice model.

In order to study the effects of LXRs on ozone exposure induced airway remodeling, some characteristics of airway structure remodeling were analyzed. The lung tissue section revealed that the alveli were enlarged and the collagen fiber was deposited around the airway after 1-week ozone stimulation. Immunohistochemistry was employed to analyze the markers of airway remodeling, including matrix metalloproteinase-9 (MMP-9) and a-smooth muscle actin (a-SMA).

T091317 has a powerful repression effect on ozone induced MMP9 production. Matrix metalloproteinases (MMPs) are mainly involved in extracellular matrix transformation and tissue repair 
[36]. Some matrix metalloproteinases, especially MMP9, may also participate in alveolar structure alterations and airway remodeling[37,38]. This is associated with the ability of MMP9 to degrade a number of extracellular matrix (ECM) proteins. The levels of MMP-9 in serum and exhaled breath condensate of COPD patients significantly increased[39, 40]. Some scholars have considered the MMP-9 concentration and the MMP-9/TIMP-1 ratio are the best predictors for emphysema in COPD patients[41, 42]. In T0901317 treated 6 week-group, the level of MMP9 was lower compared to the ozone exposure group. This suggest -LXR may have a positive significance in diminishing airway remodeling.

The a-SMA plays an important role in the proliferation and hypertrophy of airway smooth muscle cells. The present data revealed that the LXR agonist reduced the a-SMA expression in 6-week ozone-exposed mice, which is consistent with the results for MMP9. However, in mice exposed to ozone for one week, the LXR agonist didn't reduce MMP9, a-SMA, Collagen deposition and alveolar enlargement. These results indicate that the LXR agonist can alleviate the airway remodeling, but it takes a period of time for the LXR agonist to work.

ABCA1 expression significantly decreases in active smokers compared to non-smokers[43]. Foamy alveolar macrophages accumulate in the lungs of smokers $[44,45]$. Interestingly, it was reported that ozone exposure decreases the expression of ABCA1 in human bronchial epithelial cells[46].And this is closely correlated to lipid reverse transport. The model of lung inflammation results from ozone exposure bears many similarities to the cigarette smoke exposure model[17]. Therefore, the present experiment was designed to determine whether ozone exposure can damage the cholesterol reverse transport in macrophages, and whether LXR agonist T0901317 can affect the cholesterol homeostasis in ozone exposure models. But no obvious foamy macrophages were observed during the oil red $\mathrm{O}$ staining in ozone-induced mice. Given this situation, it is considered that the concentration of ozone may be too low to influence the lipid accumulation in macrophages. Furthermore, cigarette suppresses multiple genes of lipid reverse transport in cells[43] , which comprehensively contributes to foam cells. Hence, there is a need to explore these experimental conditions.

\section{Conclusions}

Asthma and COPD are characterized by chronic airway inflammation. In a previous study, the investigators have proven that in an asthma mice model, the application of T0901317 cannot reduce the inflammatory cells and Th2 cytokines in the BALF[22]. This makes it more valuable and meaningful to determine how T0901317 decreases the number of inflammatory cells and cytokines in an ozone-induced airway inflammation model. In summary, we have shown LXRs attenuate ozone induced airway inflammation and airway remodeling. Ongoing animal studies cellular research on the mechanism and biological function of LXR in the airway, will provide insights into the importance of LXR in airway disease.

\section{Abbreviations}


LXRs: Liver X receptors

IL-6: Interleukin-6

IL-8: Interleukin-8

G-CSF: Granulocyte-colony stimulating factor

MMP-9: Matrix metalloproteinase-9

COPD: Chronic obstructive pulmonary disease

a-SMA: a-smooth muscle actin

BALF: Bronchoalveolar lavage fluid

LOP: Lipid ozonation product

LPS: Lipopolysaccharide

ELISA: Enzyme linked immunosorbent assay

PBS: Ehosphate buffer saline

DMEM: Dulbecco's modified eagle medium

SUMO: Small ubiquitin-related modifier

CORO2A: Coronin 2A

hASM『Human airway smooth muscle

ABCA1: ATP-binding cassette A1 transporter

NF-kB:Nuclear factor-kappaB

TLR: Toll-like receptor

MAPK: Mitogen-activated protein kinases

MMPs: Matrix metalloproteinases

ECM: Extracellular matrix

Th2囚T-helper 2

\section{Declarations}


Acknowledgments

We would like to acknowledge all the authors for their contributions to this article and the reviewers for their helpful comments.

\section{Fund}

This study was supported by Nanjing Medical Science and technique Development Foundation (No. JQX16028) of China.

\section{Author information}

\section{Affiliations}

Fenfang Yü, Li Wang*, Nan Li,Wei Gu*, Ying Shi*

Department of Respiratory Medicine, Nanjing First Hospital, Nanjing Medical University, Nanjing 210006, China.

Lin Zheng ${ }^{\#}$, Zhifei Ma, Yu Tang

Department of Thoracic and Cardiovascular Surgery, Nanjing First Hospital, Nanjing Medical University, Nanjing 210006, China.

Xiaobing Yang Department of Pathology, Nanjing First Hospital, Nanjing Medical University, Nanjing 210006, China.

Youran Wang Department of Anesthesiology, Nanjing First Hospital, Nanjing Medical University, Nanjing 210006, China.

\#These authors contributed equally to this work.

\section{Author Contributions}

Designed this study and revised the article: GW, SY. Performed the experiment: YFF, LN, WYR. Analyzed the data: ZL, WL, YXB, TY, MZF. Draft this acticle: YFF.

\section{Corresponding author}

Ying Shi, Department of Respiration, Nanjing First Hospital, Nanjing Medical University, No. 68, Changle Road, Qinhuai District, Nanjing 210006, China. Tel.: +86-17714347735; Email: sykxss@163.com

Wei Gu, Department of Respiration, Nanjing First Hospital, Nanjing Medical University, No. 68, Changle Road, Qinhuai District, Nanjing 210006, China. Tel.: +86-25-87726231; Email: guwei_101@163.com 
The animal experiment was approved by the Animal Experiment Center of Nanjing First Hospital Affiliated to Nanjing Medical University, according to the guidelines of the Animal Care and Use Committee of the institution (license No: SYXK [Su] 2016-0006). Animal experiments were carried out in strict accordance with the provisions of the Guidance on Feeding and Use of Experimental Animals by the Ministry of Science and Technology of the People's Republic of China. All operations are performed under anesthesia, and all efforts were made to minimize the suffering.

\section{Consent for publication}

Not applicable

\section{Competing interests}

There are no competing financial interests.

\section{Availability of data and materials}

All data generated or analysed during this study are included in this published article.

\section{References}

1. Nassikas, N., et al., Ozone-related asthma emergency department visits in the US in a warming climate. Environ Res, 2020. 183: p. 109206.

2. Y, L., et al., Short-Term Exposure to Ambient Air Pollution and Asthma Mortality. American journal of respiratory and critical care medicine, 2019. 200(1): p. 24-32.

3. Medina-Ramón, M., A. Zanobetti, and J. Schwartz, The effect of ozone and PM10 on hospital admissions for pneumonia and chronic obstructive pulmonary disease: a national multicity study. Am J Epidemiol, 2006. 163(6): p. 579-88.

4. Koto, $\mathrm{H} .$, et al., Role of cytokine-induced neutrophil chemoattractant (CINC) in ozone-induced airway inflammation and hyperresponsiveness. Am J Respir Crit Care Med, 1997. 156(1): p. 234-9.

5. Pryor, W.A., G.L. Squadrito, and M. Friedman, The cascade mechanism to explain ozone toxicity: the role of lipid ozonation products. Free Radic Biol Med, 1995. 19(6): p. 935-41.

6. RM, K., et al., Induction of inflammatory mediators in human airway epithelial cells by lipid ozonation products. 1999. 160(6): p. 1934-42.

7. RM, K., et al., Activation of transcription factor IL-6 (NF-IL-6) and nuclear factor-kappaB (NF-kappaB) by lipid ozonation products is crucial to interleukin-8 gene expression in human airway epithelial cells. 2007. 22(2): p. 159-68.

8. SM, U., et al., LXR is crucial in lipid metabolism. 2005. 73(1): p. 59-63.

9. Yang, C., et al., Sterol intermediates from cholesterol biosynthetic pathway as liver $X$ receptor ligands. J Biol Chem, 2006. 281(38): p. 27816-26. 
10. Hamilton, J.P., et al., Activation of liver $X$ receptor/retinoid $X$ receptor pathway ameliorates liver disease in Atp7B(-/-) (Wilson disease) mice. Hepatology, 2016. 63(6): p. 1828-41.

11. Bedi, S., et al., Fatty acid binding profile of the liver $X$ receptor $a$. J Lipid Res, 2017. 58(2): p. 393-402.

12. Peng, D., et al., Differential anti-atherosclerotic effects in the innominate artery and aortic sinus by the liver $X$ receptor agonist T0901317. Atherosclerosis, 2009. 203(1): p. 59-66.

13. Guo, Y., et al., Synthetic High-Density Lipoprotein-Mediated Targeted Delivery of Liver X Receptors Agonist Promotes Atherosclerosis Regression. EBioMedicine, 2018. 28: p. 225-233.

14. Wang, D., et al., Synthetic LXR agonist T0901317 attenuates lipopolysaccharide-induced acute lung injury in rats. Int Immunopharmacol, 2011. 11(12): p. 2098-103.

15. Michaudel, C., et al., Functional and morphological differences of the lung upon acute and chronic ozone exposure in mice. Sci Rep, 2018. 8(1): p. 10611.

16. Sun, Z., et al., Generation of a Chronic Obstructive Pulmonary Disease Model in Mice by Repeated Ozone Exposure. J Vis Exp, 2017(126).

17. Triantaphyllopoulos, $\mathrm{K}$., et al., A model of chronic inflammation and pulmonary emphysema after multiple ozone exposures in mice. Am J Physiol Lung Cell Mol Physiol, 2011. 300(5): p. L691-700.

18. Sonett, J., et al., A critical role for $A B C$ transporters in persistent lung inflammation in the development of emphysema after smoke exposure. Faseb j, 2018. 32(12): p. fj201701381.

19. Asquith, D.L., et al., Liver $X$ receptor agonism promotes articular inflammation in murine collageninduced arthritis. Arthritis Rheum, 2009. 60(9): p. 2655-65.

20. Schulman, I.G., Liver $X$ receptors link lipid metabolism and inflammation. FEBS Lett, 2017. 591(19): p. 2978-2991.

21. Birrell, M.A., et al., Liver X Receptor Agonists Increase Airway Reactivity in a Model of Asthma via Increasing Airway Smooth Muscle Growth. 2008. 181(6): p. 4265-4271.

22. Shi, Y., et al., A Liver-X-Receptor Ligand, T0901317, Attenuates IgE Production and Airway Remodeling in Chronic Asthma Model of Mice. Plos One, 2014. 9.

23. Pichavant, M., et al., Ozone exposure in a mouse model induces airway hyperreactivity that requires the presence of natural killer T cells and IL-17. J Exp Med, 2008. 205(2): p. 385-93.

24. Zhao, Y.L., et al., Effect of total alkaloids from Alstonia scholaris on airway inflammation in rats. $\mathrm{J}$ Ethnopharmacol, 2016. 178: p. 258-65.

25. Barnes, P.J., Cellular and molecular mechanisms of chronic obstructive pulmonary disease. Clin Chest Med, 2014. 35(1): p. 71-86.

26. Hu, X., et al., Liver X Receptor Agonist TO901317 Attenuates Paraquat-Induced Acute Lung Injury through Inhibition of NF-KB and JNK/p38 MAPK Signal Pathways. Biomed Res Int, 2017. 2017: p. 4652695.

27. Miao, C.M., et al., LXRa represses LPS-induced inflammatory responses by competing with IRF3 for GRIP1 in Kupffer cells. Int Immunopharmacol, 2016. 35: p. 272-279. 
28. Kuhn, C., 3rd, et al., Airway hyperresponsiveness and airway obstruction in transgenic mice. Morphologic correlates in mice overexpressing interleukin (IL)-11 and IL-6 in the lung. Am J Respir Cell Mol Biol, 2000. 22(3): p. 289-95.

29. Im, S.S. and T.F. Osborne, Liver $x$ receptors in atherosclerosis and inflammation. Circ Res, 2011. 108(8): p. 996-1001.

30. Gao, et al., Activation of liver $X$ receptor attenuates lysophosphatidylcholine-induced IL-8 expression in endothelial cells via the NF-kappa B pathway and SUMOylation. 2016.

31. Venteclef, N., et al., GPS2-dependent corepressor/SUMO pathways govern anti-inflammatory actions of LRH-1 and LXRbeta in the hepatic acute phase response. Genes Dev, 2010. 24(4): p. 381-95.

32. Roberts, A.W., G-CSF: a key regulator of neutrophil production, but that's not all! Growth Factors, 2005. 23(1): p. 33-41.

33. Delvecchio, C.J., et al., Liver X Receptor Stimulates Cholesterol Efflux and Inhibits Expression of Proinflammatory Mediators in Human Airway Smooth Muscle Cells. 2007(6): p. 6.

34. Ito, A., et al., LXRs link metabolism to inflammation through Abca1-dependent regulation of membrane composition and TLR signaling. Elife, 2015. 4: p. e08009.

35. Thomas, D.G., et al., LXR Suppresses Inflammatory Gene Expression and Neutrophil Migration through cis-Repression and Cholesterol Efflux. Cell Rep, 2018. 25(13): p. 3774-3785.e4.

36. Klein, T. and R. Bischoff, Physiology and pathophysiology of matrix metalloproteases. Amino Acids, 2011. 41(2): p. 271-90.

37. Sampsonas, F., et al., DNA sequence variations of metalloproteinases: their role in asthma and COPD. Postgrad Med J, 2007. 83(978): p. 244-50.

38. Salib, R.J. and P.H. Howarth, Remodelling of the upper airways in allergic rhinitis: is it a feature of the disease? Clin Exp Allergy, 2003. 33(12): p. 1629-33.

39. Hao, W., et al., Inflammatory mediators in exhaled breath condensate and peripheral blood of healthy donors and stable COPD patients. Immunopharmacol Immunotoxicol, 2019. 41(2): p. 224-230.

40. Arbaningsih, S.R., et al., The The Levels of Vitamin D, Metalloproteinase-9 and Tissue Inhibitor Metalloproteinase-1 in COPD Patients, Healthy Smokers and Non-Smokers of Indonesian Citizens. 2019.

41. Uysal, P. and H.J.B. Uzun, Relationship Between Circulating Serpina3g, Matrix Metalloproteinase-9, and Tissue Inhibitor of Metalloproteinase-1 and -2 with Chronic Obstructive Pulmonary Disease Severity. 2019. 9(2).

42. Wells, J.M., et al., Elevated circulating MMP-9 is linked to increased COPD exacerbation risk in SPIROMICS and COPDGene. JCI Insight, 2018. 3(22).

43. Jubinville, É., et al., Interplay between cigarette smoking and pulmonary reverse lipid transport. Eur Respir J, 2017. 50(3).

44. Botti, T.P., et al., A comparison of the quantitation of macrophage foam cell populations and the extent of apolipoprotein E deposition in developing atherosclerotic lesions in young people: high and 
Iow serum thiocyanate groups as an indication of smoking. PDAY Research Group. Pathobiological Determinants of Atherosclerosis in Youth. Atherosclerosis, 1996. 124(2): p. 191-202.

45. Park, K.H., D.G. Shin, and K.H. Cho, Dysfunctional lipoproteins from young smokers exacerbate cellular senescence and atherogenesis with smaller particle size and severe oxidation and glycation. Toxicol Sci, 2014. 140(1): p. 16-25.

46. Speen, A.M., et al., Ozone-derived Oxysterols Affect Liver X Receptor (LXR) Signaling: A POTENTIAL ROLE FOR LIPID-PROTEIN ADDUCTS. J Biol Chem, 2016. 291(48): p. 25192-25206.

\section{Figures}
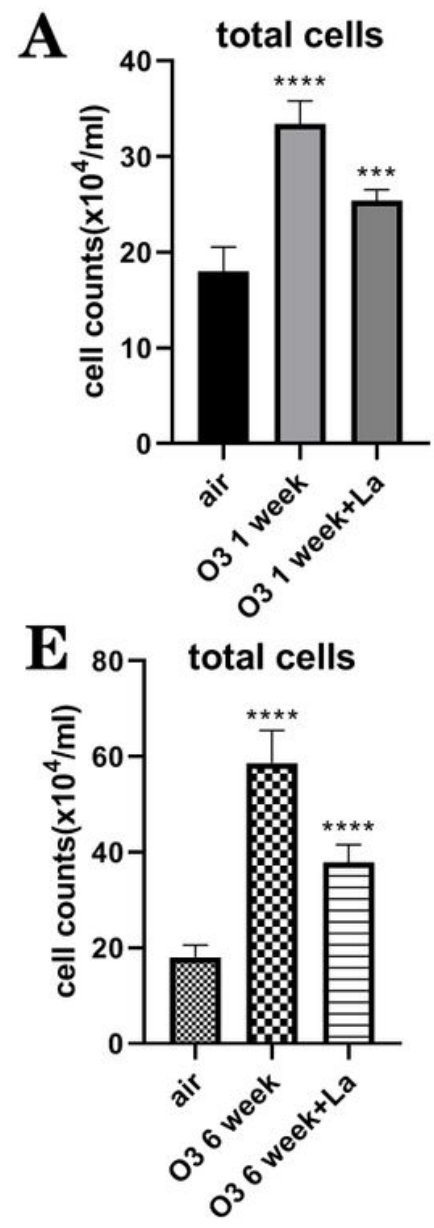
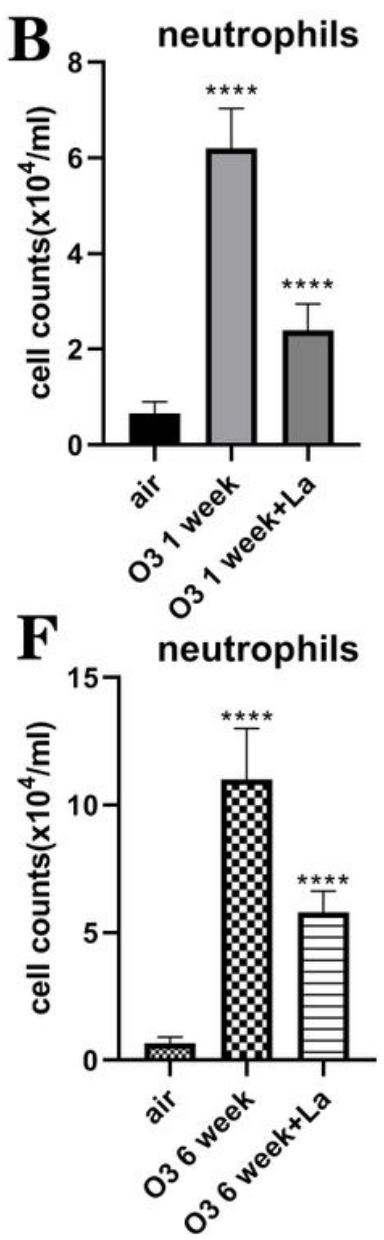
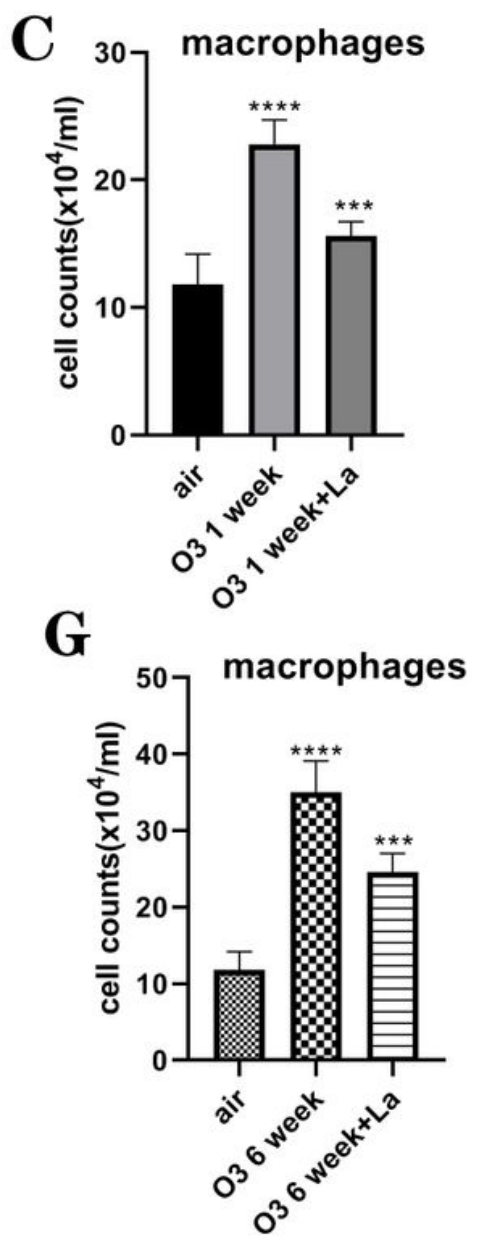
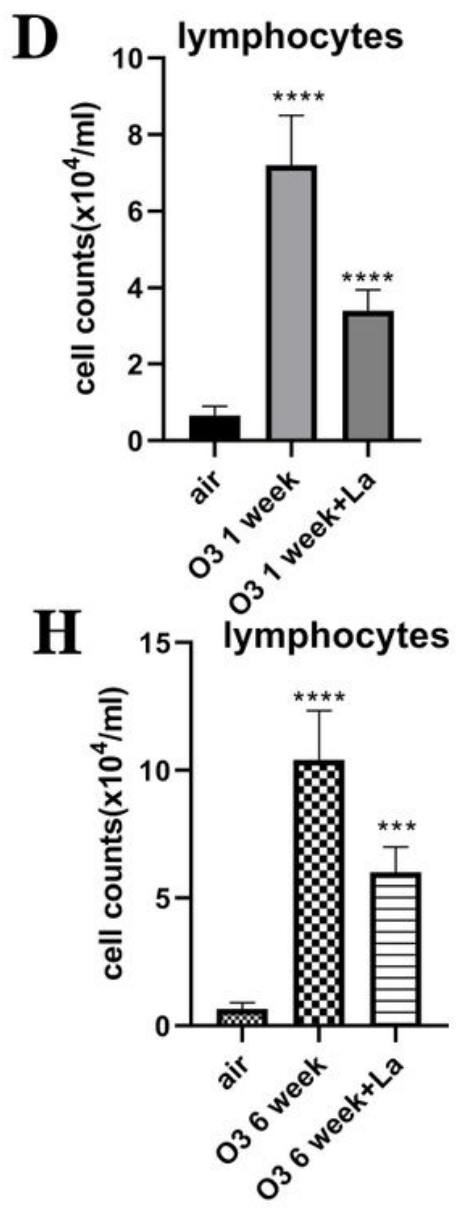

\section{Figure 1}

The LXR agonist decreases the total and differential cell counts of the BALF. Total cells were counted using a cell counting plate, and stained with Giemsa dye. Then, different cell numbers were counted at 400x magnification using a microscope. Air: air exposure only; 03 for one week: ozone exposure for one week; 03 for one week + La: ozone exposure for one week + the LXR agonist (30 ml/ Kg); 03 for six weeks: ozone exposure for six weeks; 03 for six weeks + La: ozone exposure for six weeks + the LXR agonist (30 $\mathrm{ml} / \mathrm{Kg})$. 
A

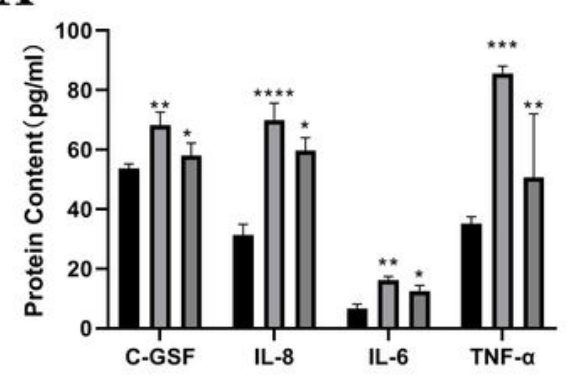

B

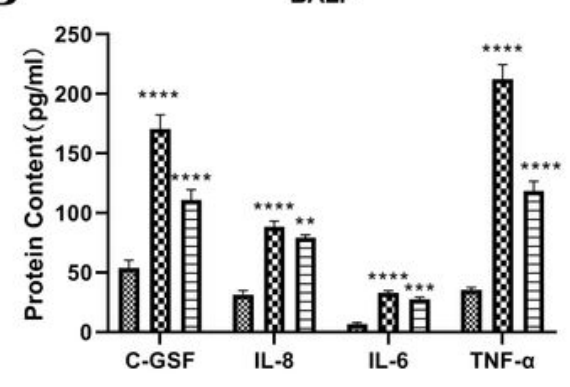

Serum

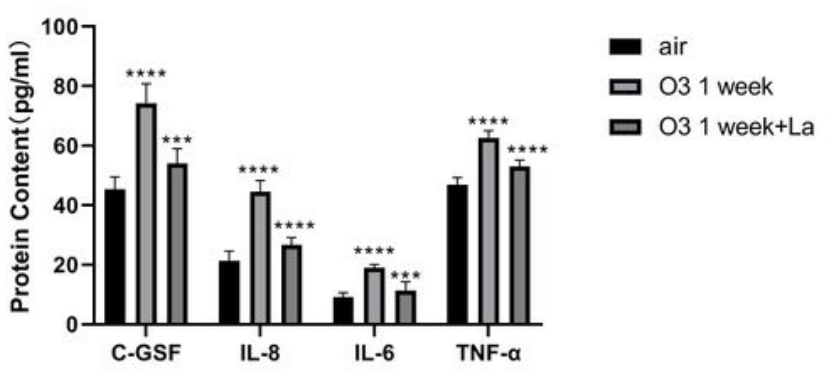

Serum

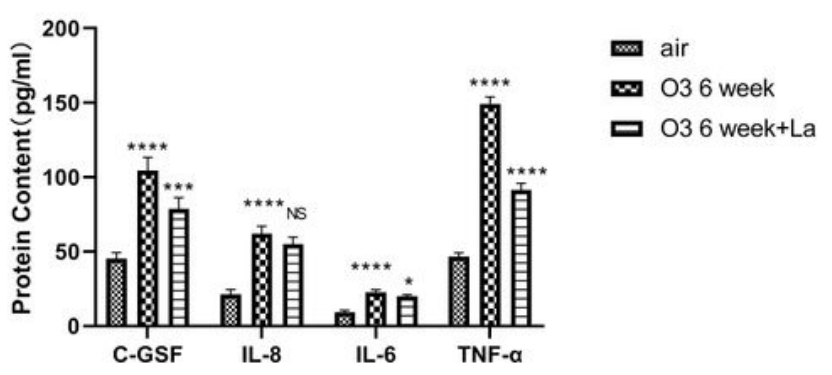

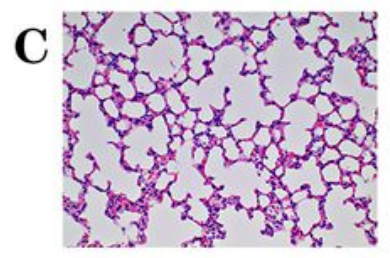
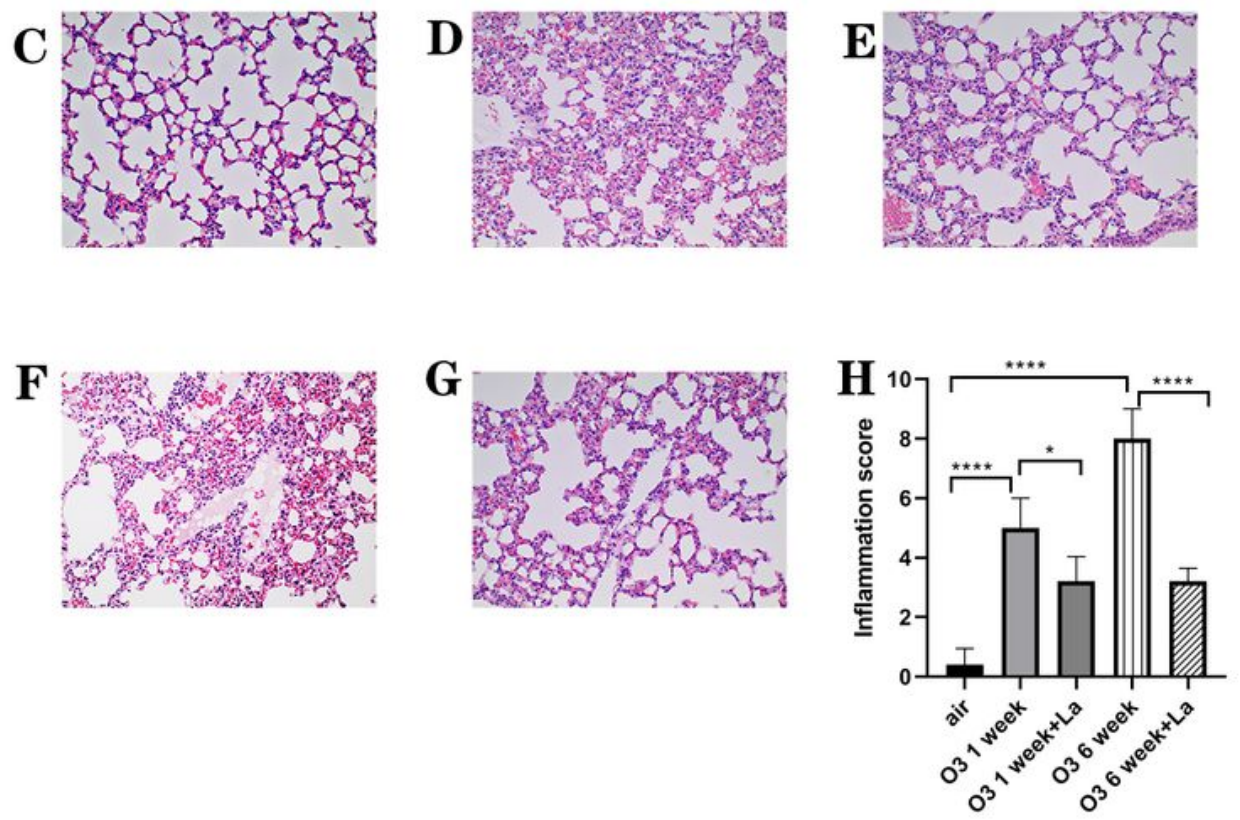

\section{Figure 2}

The LXR agonist inhibits inflammatory infiltration in lung tissues and cytokines in the BALF and serum. The levels of IL-6, IL-8, TNFa and G-CSF were determined by ELISA ( $A$ and B). A representative figure for the peribronchial lesion and alveolar lesion in lung tissues stained with the H\&E solution (C-G). The inflammation evaluation of lung tissues $(\mathrm{H})$.Air: air exposure only(C); 03 for one week(D): ozone exposure for one week; 03 for one week + La(E): ozone exposure for one week + the LXR agonist (30 ml/Kg); 03 for 
six weeks(F): ozone exposure for six weeks; 03 for six weeks + $L a(G)$ : ozone exposure for six weeks + the LXR agonist (30 ml/Kg).
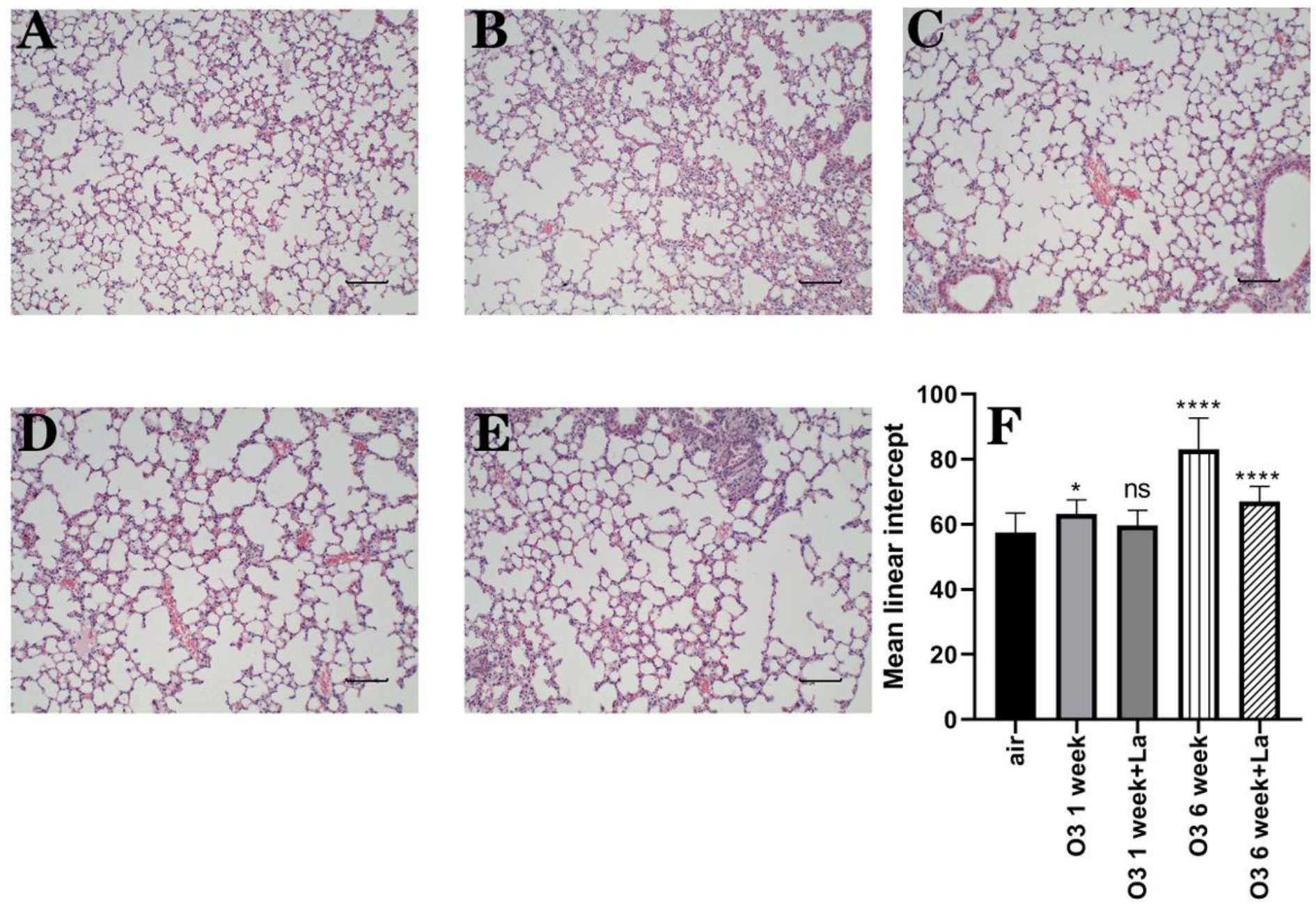

\section{Figure 3}

The LXR agonist reduces the Lm and inhibits the alveolar enlargement. (A-E) The representative H\&E stained sections from the lungs of the different groups of mice (original magnification, $\times 200)$. (F) Quantitative analysis of the lung destruction, as represented by mean linear intercept. Air: air exposure only(A); 03 for one week(B): ozone exposure for one week; 03 for one week + $\mathrm{La}(\mathrm{C})$ : ozone exposure for one week + the LXR agonist (30 ml/Kg); 03 for six weeks(D): ozone exposure for six weeks; 03 for six weeks + La(E): ozone exposure for six weeks + the LXR agonist $(30 \mathrm{ml} / \mathrm{Kg})$. 

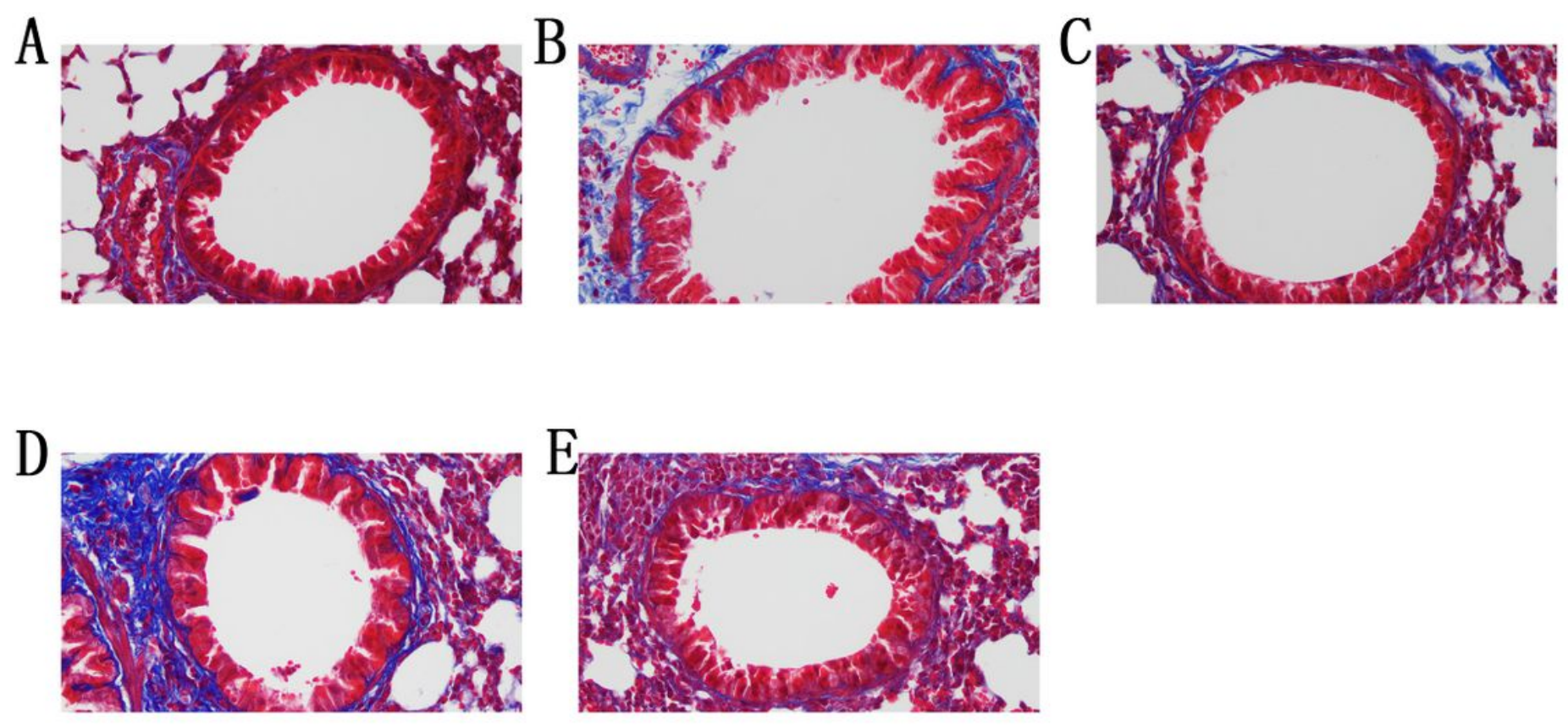

\section{Figure 4}

The LXR agonist attenuates the collagen deposition associated with ozone exposure. The representative photomicrographs demonstrate the peribronchial collagen deposition detected by Masson's trichrome staining (blue color; original magnification, $\times 400$ ). Air: air exposure only $(A)$; 03 for one week(B): ozone exposure for one week; 03 for one week + $\mathrm{La}(\mathrm{C})$ : ozone exposure for one week + the LXR agonist (30 $\mathrm{ml} / \mathrm{Kg}$ ); 03 for six weeks(D): ozone exposure for six weeks; 03 for six weeks + $\mathrm{La}(\mathrm{E})$ : ozone exposure for six weeks + the LXR agonist $(30 \mathrm{ml} / \mathrm{Kg})$. 
A
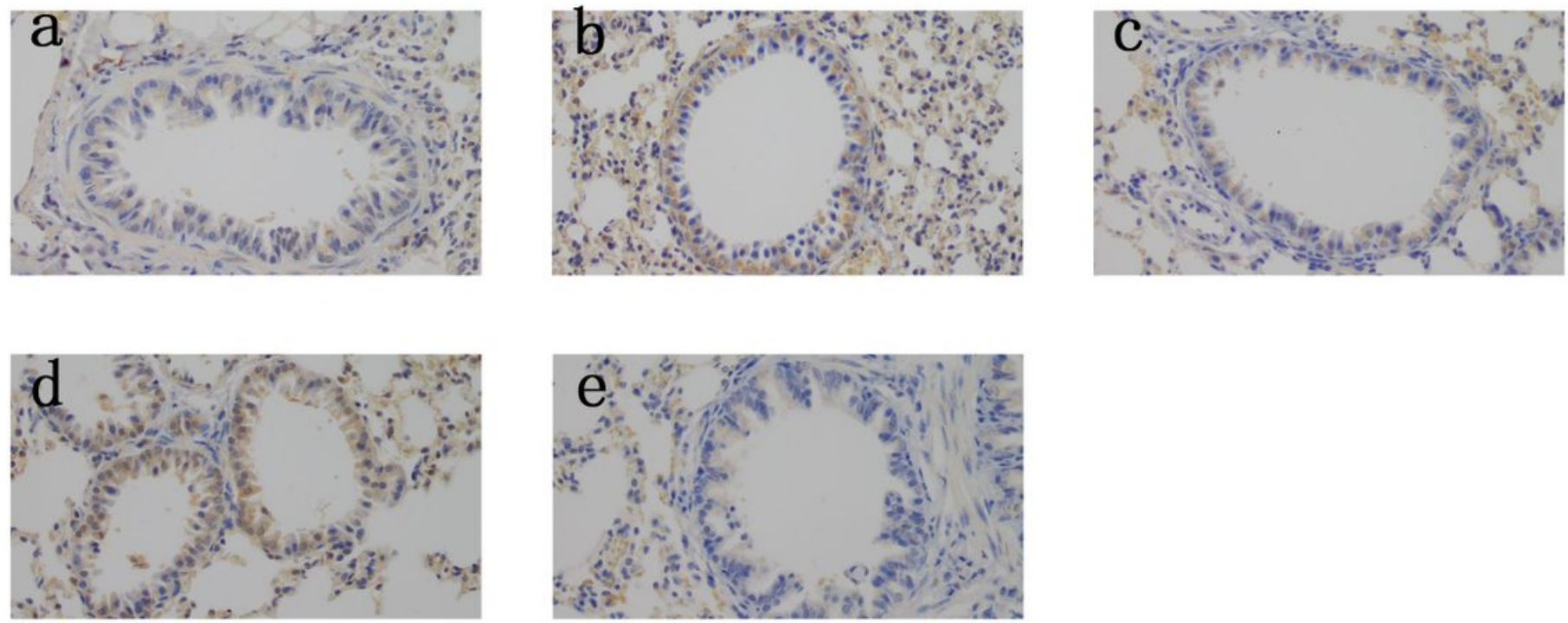

B
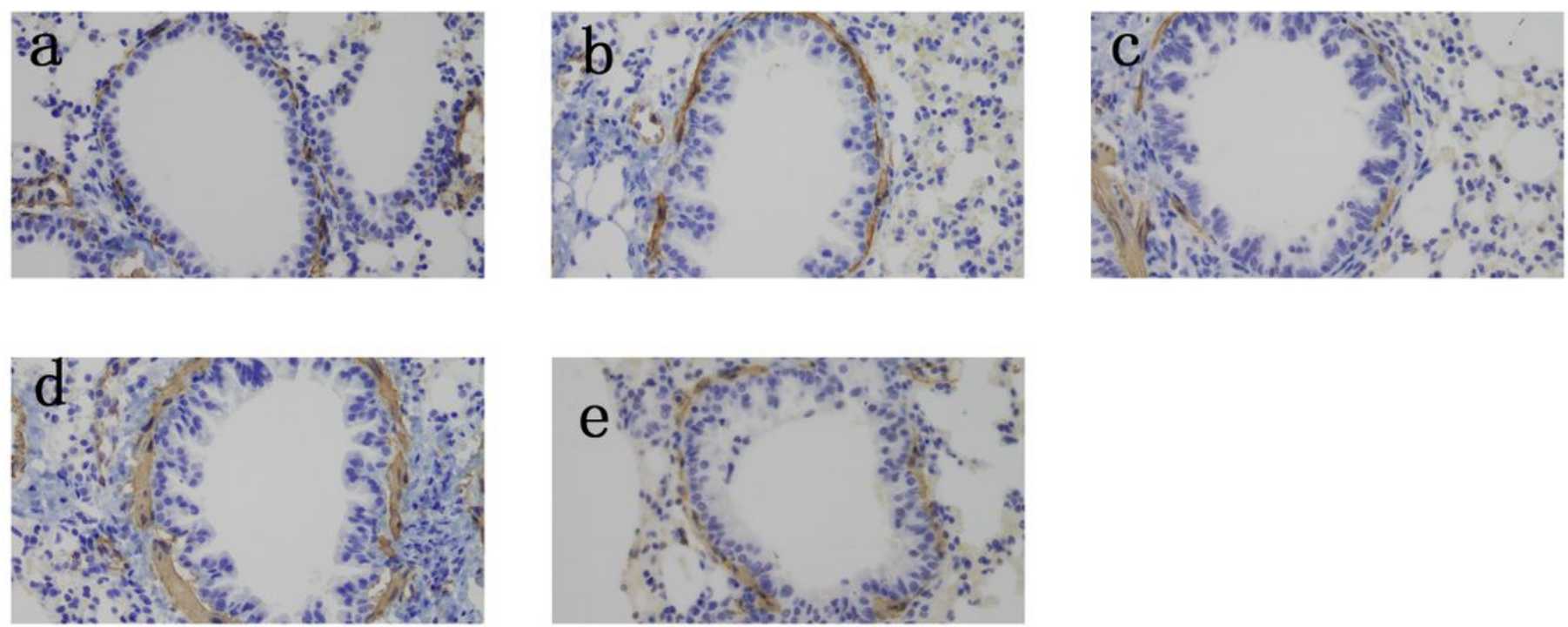

\section{Figure 5}

The LXR agonist decreases the MMP9 expression and a-SMA generation in lung tissues. The representative immunohistochemical staining (original magnification, $\times 400$ ) and mean density of MMP9 (A) and a-SMA (B) in lung tissues from the groups. Air: air exposure only(a); 03 for one week(b): ozone exposure for one week; 03 for one week + $\mathrm{La}(\mathrm{c})$ : ozone exposure for one week + the LXR agonist (30 $\mathrm{ml} / \mathrm{Kg}$ ); 03 for six weeks(d): ozone exposure for six weeks; 03 for six weeks + La(e): ozone exposure for six weeks + the LXR agonist $(30 \mathrm{ml} / \mathrm{Kg})$. 

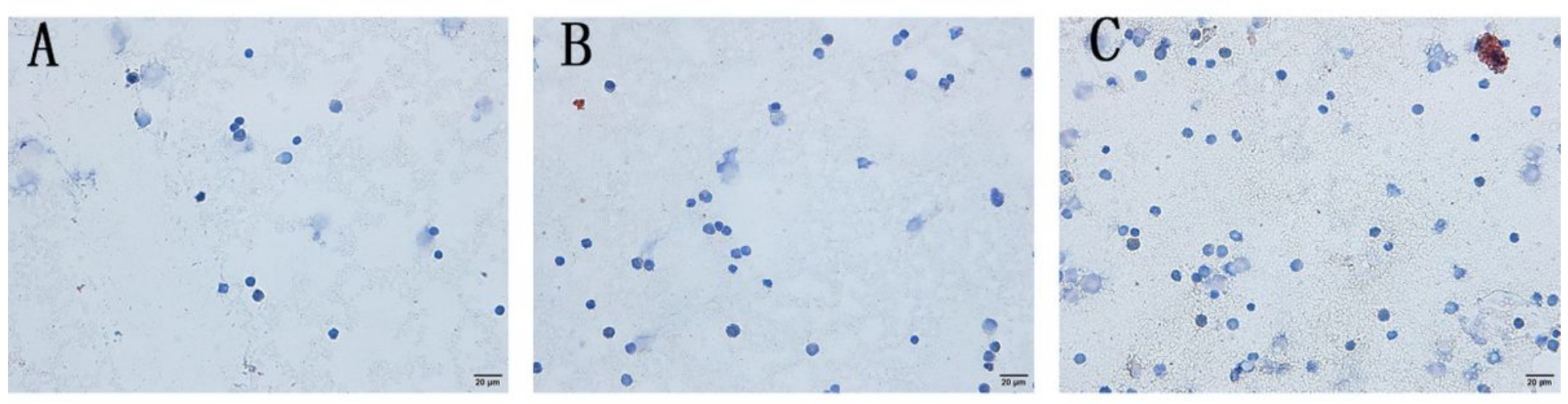

\section{Figure 6}

Ozone exposure does not lead to the formation of foam macrophages. The representative Oil red $O$ staining (original magnification, $\times 200$ ). Air: air exposure only(A); 03 for one week(B): ozone exposure for one week; 03 for six weeks(C): ozone exposure for six weeks. 